\title{
Rotation Periods of Nearby, Mid-to-late M Dwarfs from the MEarth Project
}

\author{
Elisabeth R. Newton, ${ }^{1 *}$ Jonathan Irwin, ${ }^{1}$ David Charbonneau, ${ }^{1}$ \\ Zachary K. Berta-Thomspon, ${ }^{2}$ Andrew A. West $^{3}$ \\ ${ }^{1}$ Harvard-Smithsonian Center for Astrophysics \\ *email: enewton@cfa.harvard.edu \\ ${ }^{2}$ Massachusetts Institute of Technology \\ ${ }^{3}$ Boston University
}

\begin{abstract}
Field stars provide important constraints for the late stages of stars' angular momentum evolution. We measured rotation periods ranging from 0.1 to 150 days for approximately 450 mid-to-late $\mathrm{M}$ dwarfs using photometry from the MEarth transiting planet survey. We use parallaxes, proper motions, and radial velocities to calculate galactic kinematics for these solar neighborhood $\mathrm{M}$ dwarfs. The velocity dispersions increase towards longer rotation periods, indicating that there is a relationship between rotation and age for these stars.
\end{abstract}

Keywords. stars: low-mass, stars: rotation, stars: kinematics and dynamics

\section{Introduction}

Rotation is one of the few directly measurable stellar properties and encodes the history of a star's angular momentum evolution. Late-time angular momentum loss is governed by stellar winds, which depend on the stars' magnetic field topologies. Stars ages, stellar winds, and magnetic properties are important for the detection and characterization of their planetary systems.

Field stars provide important constraints for the late stages of angular momentum evolution. These data are particularly important for fully-convective stars, which continue to undergo substantial rotational evolution at field ages. Irwin et al. (2011) contributed many of the currently-available measurements for fully-convective stars, with rotation periods for $41 \mathrm{M}$ dwarfs from the MEarth Project (Berta et al., 2012; Irwin et al., 2015). Newton et al. (submitted) extended this analysis to the full sample of $\mathrm{M}$ dwarfs observed by MEarth. Our team has gathered supplementary observations of these stars: Dittmann et al. (2014) measured parallaxes for 1507 of the MEarth targets using MEarth photometry and Newton et al. (2014) obtained low-resolution near-infrared spectra of 447 MEarth targets, measuring their absolute radial velocities and estimating their metallicities. These data enable further studies of the stars with rotation periods from MEarth.

\section{Data and analysis}

The MEarth Project is an all-sky survey searching for planets transiting 3000 nearby, mid-to-late M dwarfs. MEarth-North has been operational since September, 2008 and MEarth-South was commissioned in January, 2014. Each observatory consists of eight $40 \mathrm{~cm}$ telescopes on German Equatorial Mounts, which use red-optical filters.

We searched for stellar variability in all MEarth targets observed as of April 2015, simultaneously fitting for systematics and a sinusoidal rotational modulation (Irwin et al., 2011). We compared the sinusoidal model to a null hypothesis, testing rotation periods 

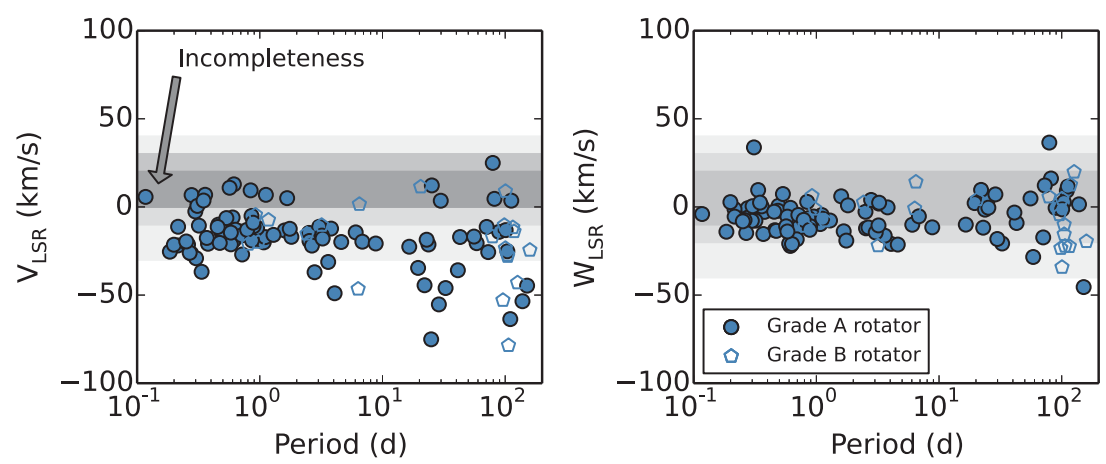

Figure 1. Individual components of space velocity as a function of measured photometric rotation period for stars with $M<0.25 M_{\odot}$. Gray shaded regions indicate the velocities with higher incompleteness due to our proper motion limits; contours are $5-20 \%$.

from 0.1 to 1000 days. We selected the best-fitting period and visually inspected each light curve. We qualitatively assessed the candidate period, and identified the approximately 450 stars with clear modulations as rotators. Those with unambiguous variability were classified Grade A, and those in which we were less certain were classified as Grade B.

\section{Rotation and galactic kinematics}

The rotation periods $(P)$ we detected range from 0.1 days to 150 days. We highlight the existence of slow rotators with high amplitudes of variability. These slowly-rotating stars have enough spots to produce peak-to-peak amplitudes of a percent or more.

We calculated $U V W$ velocities for 660 stars in our sample. The substructure seen in the kinematics of higher-mass stars (e.g. Nordstrom et al., 2004) is seen amongst the M dwarfs, including the dynamically-created Hyades supercluster. Following the methods of Bensby et al. (2003), our rotators have high probabilities of belonging to the thin disk, which differs significantly from the distribution for all $\mathrm{M}$ dwarfs in our sample.

There is clear evidence for a rotation-age relation in all three velocity components (Fig. 1). The velocity dispersions increase with rotation period, as expected for a dynamicallyheated population, and we see evidence of asymmetric drift in $V$. We use $\sigma_{W}$ and the Aumer \& Binney (2009) age-velocity relationship to estimate the ages for different populations of rotators. We estimate that $\mathrm{M}$ dwarfs with $M<0.25 M_{\odot}$ and $P<10$ days are $<2$ Gyr old and those with $70<P<150$ days are $4-8$ Gyr.

\section{References}

Aumer, M. \& Binney, J. J. 2009, MNRAS, 397, 1286

Berta, Z. K., Irwin, J., Charbonneau, D., Burke, C. J., \& Falco, E. E. 2012, AJ, 144, 145

Bensby, T., Feltzing, S., \& Lundström, I. 2003, AESA, 410, 527

Dittmann, J. A., Irwin, J. M., Charbonneau, D., \& Berta-Thompson, Z. K. 2014, ApJ, 784, 156 Irwin, J. M., Berta-Thompson, Z. K., \& Charbonneau, D., et al. 2015, in 18th Cambridge Workshop on Cool Stars, Stellar Systems, and the Sun, 18, 767

Irwin, J., Berta, Z. K., \& Burke, C. J., et al. 2011, ApJ, 727, 56

Newton, E. R., Charbonneau, D., Irwin, J., et al. 2014 AJ, 147, 20

Newton, E. R., Irwin, J., Charbonneau, D., et al. submitted to ApJ

Nordström, B., Mayor, M., \& Andersen, J., et al. 2004, A\&A, 418, 989 